\title{
Avaliação da cinética de secagem da fibra do sisal e do uso das fibras na adsorção de corante têxtil sintético
}

\author{
Evaluation of sisal fiber drying kinetics and \\ fiber use in synthetic textile dye adsorption
}

Fellipe Farias Crispiniano ${ }^{1}$, Cinthia Sany França Xavier ${ }^{1}$, Fernando Fernandes Vieira ${ }^{1}$, Marcelo Maia de Almeida ${ }^{1}$

\footnotetext{
${ }^{1}$ Universidade Estadual da Paraíba, Programa de Pós-Graduação em Ciência e Tecnologia Ambiental, Rua Juvêncio Arruda, S/N - CEP: 58429-600 Campus Universitário, Bodocongó, Campina Grande, Paraíba, Brasil. e-mail: fellipetecinfo@gmail.com, cinthiasany@gmail.com,fernando@uepb.edu.br,marcelo_maia2000@yahoo.com
}

\section{RESUMO}

Os resíduos agroindustriais são materiais alternativos de baixo custo e possuem um grande potencial como adsorvente de poluentes. Partindo desse princípio, o presente estudo avaliou o potencial da fibra do sisal como adsorvente de corante têxtil. Antes de proceder com os ensaios de adsorção, foram realizados estudos de cinética de secagem da fibra do sisal nas temperaturas de $60{ }^{\circ} \mathrm{C}$ e $80{ }^{\circ} \mathrm{C}$. Na ocasião, o menor tempo do equilíbrio de secagem foi alcançado em $180 \mathrm{~min}$ na temperatura de $80^{\circ} \mathrm{C}$. Com o intuito de alcançar melhores resultados, os dados de secagem foram ajustados aos modelos de Page, Henderson e Pabis, Newton O'Callaghan, Logarítmico e Midilli. Os resultados mostraram que o modelo de Midilli mostrou-se o mais adequado para o processo em ambas as temperaturas. Quanto aos ensaios de adsorção, eles foram realizados em banho finito em que, se utilizou $25 \mathrm{ml}$ da solução com corante, $0,25 \mathrm{~g}$ da fibra do sisal e agitação de 120 $\mathrm{rpm}$. No estudo da adsorção, aplicou-se um planejamento fatorial $2^{3}$ para avaliar a influência da concentração do corante, da temperatura de secagem e do tempo de contato no processo de adsorção. Os resultados das análises estatística do planejamento fatorial $2^{3}$, mostraram que todas as variáveis de controle estudadas influenciam o processo de adsorção. Os maiores valores das variáveis $q\left(\mathrm{mg} \cdot \mathrm{g}^{-1}\right.$ ) e Remoção (\%) foram 3,23 mg.g ${ }^{1}$ e $64,52 \%$ respectivamente. Partindo dos efeitos significativos, foi possível conceber modelos empíricos para as variáveis dependentes, já que, todas apresentaram significância estatística para um nível de confiança de $95 \%$. Os coeficientes de determinação para os modelos empíricos foram, 99,64\% e 96,44\% respectivamente. Diante dos resultados obtidos, as fibras do sisal se mostraram como um excelente adsorvente alternativo, para o tratamento de águas residuárias, contendo corante têxtil.

Palavras-chave: sisal; secagem; adsorção; corante.

\begin{abstract}
Agroindustrial waste is a low cost alternative material and has great potential as a pollutant adsorbent. Based on this principle, the present study evaluated the potential of sisal fiber as a textile dye adsorbent. Prior to the adsorption tests, drying kinetics studies of the sisal fiber were performed at temperatures of $60^{\circ} \mathrm{C}$ and $80{ }^{\circ} \mathrm{C}$. At the time, the shortest drying balance time was reached in $180 \mathrm{~min}$ at $80{ }^{\circ} \mathrm{C}$. In order to achieve better results, the drying data were adjusted to Page, Henderson and Pabis, Newton O'Callaghan, Logarithmic and Midilli models. The results showed that the Midilli model was the most suitable for the process at both temperatures. As for the adsorption tests, they were performed in a finite bath in which $25 \mathrm{ml}$ of the dye solution, $0.25 \mathrm{~g}$ of sisal fiber and agitation of $120 \mathrm{rpm}$ were used. In the adsorption study, a $2^{3}$ factorial design was applied to evaluate the influence of dye concentration, drying temperature and contact time on the adsorption process. The results of the statistical analysis of the $2^{3}$ factorial design showed that all control variables studied influence the adsorption process. The highest values of the variables $q$ (mg.g $\left.{ }^{-1}\right)$ and Removal (\%) were $3.23 \mathrm{mg} \cdot \mathrm{g}^{-1}$ and $64.52 \%$ respectively. From the significant effects, it was possible to conceive empirical models for the dependent variables, since all presented statistical significance for a confidence level of $95 \%$. The determination coefficients for the empirical models were $99.64 \%$ and $96.44 \%$ respectively. In view of the results obtained, the sisal fibers proved to be an excellent alternative adsorbent, for the treatment of wastewater, containing textile dye.
\end{abstract}


Keywords: sisal; drying; adsorption; dye.

\section{INTRODUÇÃO}

A indústria têxtil no Brasil possui um papel muito importante no que diz respeito à geração de riquezas e de empregos. Essa atividade se consolidou fortemente tornando o País um importante produtor mundial de artigos têxteis. Todavia, essa atividade gera diversos problemas ambientais como, por exemplo, a geração de resíduos sólidos e líquidos oriundos dos processos industriais [1].

A exemplo, os processos de tingimento que consomem um grande volume de água, levando em conta todas as etapas de beneficiamento, sendo que $80 \%$ desse volume é descartado como efluente. Esses efluente possuem altas concentrações de sais, sólidos suspensos, e principalmente cor [2].

Várias técnicas são utilizadas no tratamento de águas residuais tais como: adsorção, coagulação, processos oxidativos e separação por membranas. Dentre os métodos mais eficientes destaca-se a adsorção por carvão ativado. Os adsorventes são compreendidos como sendo substâncias sólidas, porosas e que apresentam uma área superficial elevada onde ocorre a adsorção do adsorvato, na qual a substância química (adsorvato) se acumula na superfície do adsorvente [3].

Segundo MARCHI et al. [4] a adsorção é considerada como um fenômeno físico de superfície onde uma substância é removida de uma fase e concentrada em outra. O material removido é intitulado de adsorvato e o material onde o fenômeno de adsorção ocorre é chamado de adsorvente.

As forças envolvidas no processo de adsorção podem ser físicas (fisissorção) ou química (quimissorção). A adsorção física envolve forças fracas entre as moléculas do adsorvato e a superfície do adsorvente, na qual essa força intermolecular é atribuída, principalmente, à interação de Van der Waals [5]. Na adsorção química as moléculas ou íons se unem ao adsorvente por ligações químicas, normalmente ligações covalentes, e tendem a se depositar em sítios onde propiciem o maior número de coordenação [6]

Nos últimos 20 anos, materiais fibrosos naturais como: Juta, Cânhamo, Sumáuma e sisal (Agave sisala$n a)$, tornaram-se um dos bioadsorventes mais econômicos e de baixo custo. Isso é devido às suas propriedades, como: morfologia da superfície, área superficial, ampla porosidade, alta permeabilidade a gás e pequenos poros inter-fibrosos [7].

De acordo com RODRIGUES et al. [8], a secagem deve ser uma das primeiras etapas do processamento quando se utiliza resíduos lignocelulósicos, essencialmente para os que necessitam ser transportados ou armazenados. Esse processo também é importante para reduzir a atividade da água, tendo como objetivo a diminuição da velocidade de reações de decomposição do material, da proliferação de microrganismos, de processos químicos, escurecimento por oxidação e atividade enzimática.

Segundo GONELI et al. [9], a secagem consiste em um processo complexo, na qual a transferência de calor e massa entre o ar e o produto a ser seco estão envolvidos. Com o aumento da pressão parcial de vapor, provocado pelo aumento da temperatura, o material tem seu teor de umidade reduzido.

$\mathrm{O}$ estudo da secagem de materiais, também tem como objetivo promover um menor gasto de energia durante o processo. MENEZES et al. [10] afirma que a análise econômica do processo, visa determinar as melhores condições de secagem onde a amostra possa apresentar a menor umidade de equilíbrio em curto período de tempo para que possa ser armazenada sem que o material perca suas características.

Na literatura, existem três tipos de modelos de secagem que são muito usados na modelagem dos dados experimentais de cinética de secagem de produtos agrícolas, são eles: modelos teóricos, os modelos semiempíricos e empíricos [9].

Diante do exposto, objetivou-se no presente trabalho, avaliar as melhores condições de secagem para a fibra do sisal por meio do estudo da cinética de secagem e analisar a influência das temperaturas de secagem em estufa com circulação de ar, concentração do corante e tempo de contato no processo de adsorção do corante sintético Tupy 16 Bordô.

\section{MATERIAIS E MÉTODOS}

\subsection{Preparo do adsorvente}

As fibras do sisal foram cortadas em pequenos pedaços, aproximadamente entre 1 e $2 \mathrm{~cm}$. Após o corte o material foi lavado com a finalidade de remover as impurezas presentes nas fibras. 


\subsection{Determinação da curva de secagem}

Determinou-se a umidade inicial do sisal em estufa (Modelo TE - 393/2) a $105{ }^{\circ} \mathrm{C} \pm 5{ }^{\circ} \mathrm{C}$ por um período de 24 horas. Para o levantamento dos dados cinéticos necessários ao estudo da secagem, foram realizados experimentos de secagem em duas temperaturas $\left(60^{\circ} \mathrm{C}\right.$ e $\left.80{ }^{\circ} \mathrm{C} \pm 2{ }^{\circ} \mathrm{C}\right)$, com o auxílio de uma estufa com recirculação de ar (modelo 400/ND) com fluxo de ar de 1,0 m.s ${ }^{-1}$. Os experimentos foram realizados em triplicata, nos quais foram utilizadas bandejas de alumínio contendo $200 \mathrm{~g}$ da fibra do sisal. Nos primeiros $60 \mathrm{~min}$, de experimento, as bandejas foram pesadas em balança semianalítica (modelo BK 2000) em intervalos de tempo de 5 min, até completar uma hora. Em seguida, o intervalo de pesagem passou a ser a cada 10 min, até que se completasse duas horas. Passados duas horas, as pesagens passaram a ser a cada $15 \mathrm{~min}$, até que se completasse três horas. Desse ponto em diante, as pesagens passaram a ser a cada $30 \mathrm{~min}$, até se obter massa constante.

Com os dados de secagem, foi possível construir as curvas de secagem, que é obtida por meio da umidade em função do tempo. A razão de umidade e taxa de secagem são obtidas pelas Equações 1 e 2, respectivamente:

$$
\begin{gathered}
R U=\frac{X_{t}-X_{e}}{X_{i}-X_{e}} \\
\operatorname{Taxa}=\frac{d X_{(b . s)}}{d t} \cong \frac{\Delta X}{\Delta t}
\end{gathered}
$$

Onde $R U$ é a razão de umidade do sisal; $X_{\mathrm{t}}$ é o teor de água do produto no tempo; $X_{\mathrm{e}}$ é o teor de água do sisal no equilíbrio e $X_{\mathrm{i}}$ é o teor de água inicial do produto. A taxa de secagem é obtida por meio da derivada da curva de secagem. Numericamente a taxa pode ser calculada pela diferença entre as umidades no intervalo considerado [11].

Após essa etapa as fibras do sisal foram trituradas com auxílio de um moinho de facas modelo MA 04 MARCONI, utilizado a malha mesh 20 para se obter um tamanho uniforme. Segundo Rodrigues et al. (2016) a etapa de moagem é necessária para aumentar a área de superficial, através da redução do tamanho de partículas e uniformizar o tamanho das partículas para melhorar a eficiência dos tratamentos subsequentes.

Posteriormente o material foi lavado com água destilada, com o objetivo de remover a cor liberada pelas fibras trituradas. Após a lavamgem o material foi seco, a $60{ }^{\circ} \mathrm{C}$ e $80^{\circ} \mathrm{C}$ por 24 horas, e armazenado em caixa térmica de isopor.

\subsubsection{Modelagem da cinética de secagem}

Os dados experimentais de razão de umidade, em função do tempo de secagem, foram submetidos à análise por meio de regressão não-linear utilizando o método de Levenberg-Marquardt, com critério de convergência de 0,000001 . O critério de escolha do modelo foi feito com base nos maiores valores do coeficiente de determinação $\left(\mathrm{R}^{2}\right)$ e o teste $\mathrm{F}$, obtido na análise de variância (ANOVA). O software utilizado para a realização das análises estatísticas foi o Statistica versão 12. A Tabela 1 apresenta os modelos aos quais os dados de secagem foram ajustados para cada tempo.

Tabela 1- Modelos matemático de cinética de secagem de produtos agrícolas.

\begin{tabular}{lc}
\hline DESIGNAÇÃO DOS MODELOS & MODELOS \\
\hline Page & $\mathbf{R U}=\boldsymbol{e}^{\left(-\boldsymbol{k} \cdot \boldsymbol{t}^{\boldsymbol{n}}\right)}$ \\
Henderson e Pabis & $\mathbf{R U}=\boldsymbol{a} \cdot \boldsymbol{e}^{-\boldsymbol{k} . \boldsymbol{t}}$ \\
Newton O'Callaghan & $\mathbf{R U}=\boldsymbol{e}^{(-\boldsymbol{k} \cdot \boldsymbol{t})}$ \\
Logarítmico & $\mathbf{R U}=\boldsymbol{a} \cdot \boldsymbol{e}^{-\boldsymbol{k} . \boldsymbol{t}}+\boldsymbol{b}$ \\
Midilli & $\mathbf{R U}=\boldsymbol{a} \cdot \boldsymbol{e}^{-\boldsymbol{k} \boldsymbol{t}^{\boldsymbol{n}}+\boldsymbol{b} . \boldsymbol{t}}$
\end{tabular}

Onde: $n, a$ e $b$ são as constantes adimensionais dos modelos; $k\left(\min ^{-1}\right)$ é a constate de secagem; $t$ (min) é o tempo de secagem. 


\subsection{Planejamento fatorial $2^{3}$}

O fenômeno de adsorção é influenciado por vários fatores. Para compreender esse fenômeno, o estudo estatístico se faz necessário na otimização dos experimentos para que se identifique quais efeitos são significativos para o processo de adsorção. Portanto, utilizou-se um planejamento em dois níveis, com três repetições sem ponto central, tendo como variáveis de controle a concentração do corante $\left(25 \mathrm{mg} . \mathrm{L}^{-1}\right.$ e $\left.50 \mathrm{mg} . \mathrm{L}^{-1}\right)$, temperatura de secagem $\left(60{ }^{\circ} \mathrm{C}\right.$ e $\left.80^{\circ} \mathrm{C}\right)$ e tempo de contato $(30 \mathrm{~min}$ e $60 \mathrm{~min})$.

\subsubsection{Preparo da solução estoque}

Foi preparada uma solução com o corante sintético (Tupy 16 Bordô), com concentração de $1000 \mathrm{mg} \cdot \mathrm{L}^{-1}$. A solução foi armazenada em recipiente cor âmbar para posterior utilização. As leituras da concentração, foram realizadas utilizando um espectrofotômetro UV - VIS no comprimento de $\lambda$ máx $=520 \mathrm{~nm}$. A faixa linear de trabalho foi de 0 a $100 \mathrm{mg} \cdot \mathrm{L}^{-1}$.

\subsubsection{Processo de adsorção em banho finito}

No ensaio de adsorção em banho finito, foram utilizados $0,25 \mathrm{~g}$ do adsorvente in natura, obtido na secagem, e uma alíquota de $25 \mathrm{~mL}$ da solução com o corante. O material foi pesado em balança analítica (modelo Fa2104n com precisão de $0,1 \mathrm{mg}$ ). Os erlenmeyers $(125 \mathrm{~mL})$ contendo o adsorvente e o corante, foram submetidos a agitação de $120 \mathrm{rpm}$, em mesa agitadora orbital (Marca Nova técnica), até o término do tempo de contato determinado pelo planejamento experimental $2^{3}$. Em seguida as amostras foram centrifugadas sob rotação de 2500 rpm por 30 minutos. Após a centrifugação as amostras foram analisadas em espectrofotômetro UV - Vis em $\lambda$ máx $=520 \mathrm{~nm}$.

O cálculo da quantidade de corante removida por unidade de massa de adsorvente e a eficiência na remoção, foram calculadas utilizando as Equações 3 e 4, respectivamente:

$$
\begin{gathered}
q=\frac{V *\left(C_{i}-C_{t}\right)}{m} \\
\operatorname{Remoç} \tilde{a} o(\%)=\frac{\left(C_{i}-C_{t}\right) * 100}{C_{i}}
\end{gathered}
$$

Onde: $q$, é a quantidade de corante removida por unidade de massa de adsorvente (mg. $\left.\mathrm{g}^{-1}\right) ; \mathrm{V}$, é o volume da solução com o corante $(\mathrm{L}) ; C_{\mathrm{i}}$, é a concentração inicial do corante $\left(\mathrm{mg} . \mathrm{L}^{-1}\right) ; C_{\mathrm{t}}$, é a concentração no tempo; $m$, é a massa de adsorvente (mg.L $\left.\mathrm{L}^{-1}\right)$; Remoção (\%) , é a eficiência na remoção de corante.

\section{RESULTADOS E DISCUSSÃO}

A umidade inicial do sisal foi obtida em estufa a $105^{\circ} \mathrm{C}$ por um período de 24 horas. Nessa etapa foi possível determinar a quantidade de água contida inicialmente no sisal, que representa $68 \%$ base úmida (b.u.) de sua massa total. Por meio das curvas de secagem e da taxa de secagem, é possível analisar a influência da velocidade do fluxo de ar e da temperatura na secagem da fibra do sisal.

Na Figura 1, temos as curvas de cinética da secagem do sisal nas temperaturas de $60{ }^{\circ} \mathrm{C}$ e $80^{\circ} \mathrm{C}$. 


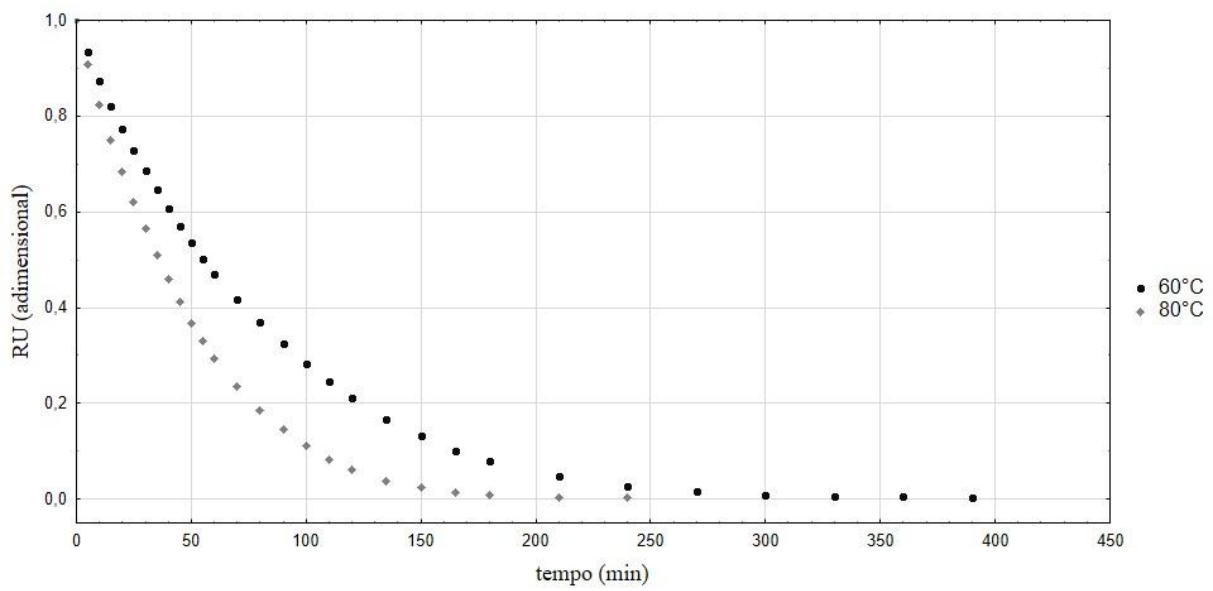

Figura 1: Curva da cinética da secagem do sisal em estufa de recirculação de ar nas temperaturas de $60{ }^{\circ} \mathrm{C}$ e $80{ }^{\circ} \mathrm{C}$.

Pode-se observar, pelo gráfico da cinética de secagem, que a fibra do sisal seca na temperatura de $60{ }^{\circ} \mathrm{C}$, com fluxo ar de secagem de $1 \mathrm{~m} . \mathrm{s}^{-1}$, atingiu a umidade de equilíbrio após um período de $300 \mathrm{~min}$, em contra partida o material seco na temperatura de $80{ }^{\circ} \mathrm{C}$, levou $180 \mathrm{~min}$ para atingir a umidade de equilíbrio. Nota-se que o aumento da temperatura do ar, acelerou a perda de umidade das fibras do sisal, que fez com que as fibras alcançassem o equilíbrio rapidamente. Pode-se observar a influência da temperatura no processo de secagem, uma vez que o aumento dessa variável resulta na diminuição da umidade e, consequentemente, na redução do tempo de secagem.

O tempo de secagem é dependente da temperatura de secagem, pois, em temperaturas mais elevadas o tempo de secagem se torna menor. ARAÚJO et al. [12] no estudo da cinética de secagem, observou que, para todas as temperaturas analisadas, a razão de umidade reduziu-se rapidamente no início e, posteriormente, houve uma diminuição progressiva da umidade à medida que o tempo de secagem aumentava.

Segundo BOTELHO et al. [13] o aumento da temperatura dentro da estufa, proporciona o aumento do potencial de secagem do ar e da transferência de calor para o material, fazendo com que a velocidade de migração do vapor de água do interior do material para sua superfície seja maior.

Isso ocorre devido a maior taxa de remoção de água no interior do produto, que é influenciada pela maior transferência de energia na forma de calor [14].

$\mathrm{Na}$ Figura 2, são representadas as curvas de taxa de secagem, que foram obtidas por meio da derivada da umidade em base seca em relação ao tempo, para o material seco nas temperaturas de $60{ }^{\circ} \mathrm{C}$ e $80{ }^{\circ} \mathrm{C}$.

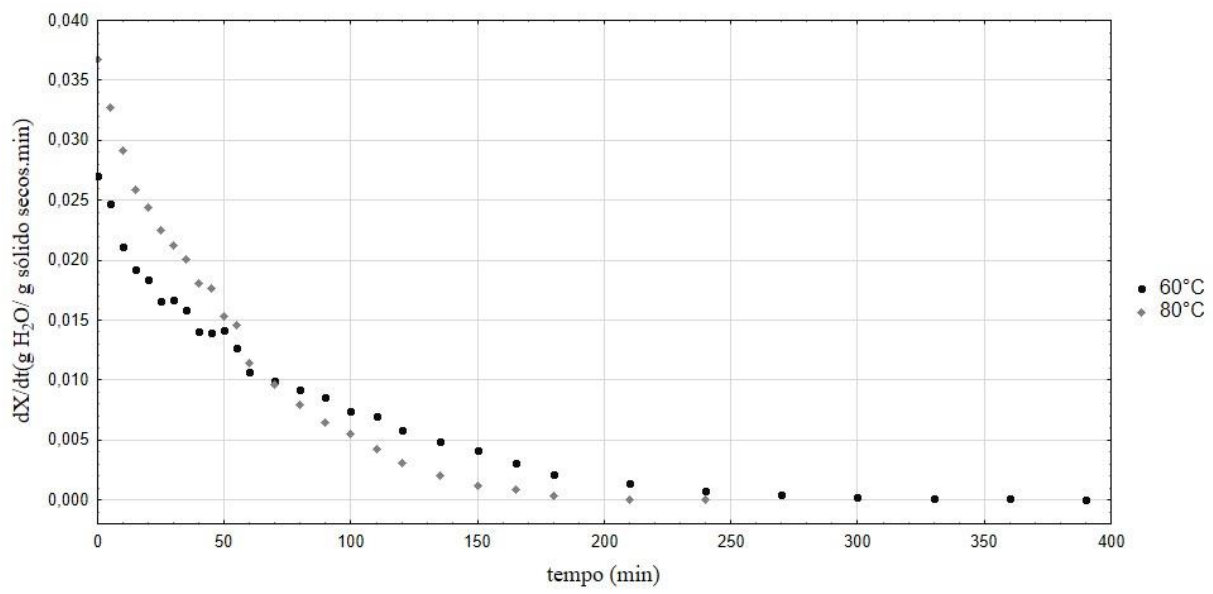

Figura 2: Curva da taxa de secagem nas temperaturas $60{ }^{\circ} \mathrm{C}$ e $80^{\circ} \mathrm{C}$.

Pode-se observar que a o material seco na temperatura de $80{ }^{\circ} \mathrm{C}$, possui uma taxa de secagem maior, que o material seco a $60{ }^{\circ} \mathrm{C}$, portanto a perda de umidade nessas condições e mais rápida.

Constatou-se que as curvas da taxa de secagem, da fibra do sisal, apresentam apenas os períodos de taxa 
decrescente. De acordo com PARK et al. [11] neste período a resistência interna do material passam a predominar e a taxa de secagem diminui gradativamente.

ARAUJO et al. [15] no estudo da cinética de secagem dos frutos de amendoim, identificou que no processo de secagem ocorreu predominantemente o período de taxa decrescente, que está associado com a maior resistência a transferência de água no interior do produto, o que torna a taxa de evaporação superficial superior à taxa de reposição de água na superfície do produto.

No período de taxa decrescente a difusão é o mecanismo físico dominante que rege o movimento da umidade no material. Geralmente esse comportamento é característico de materiais biológicos, no qual, é possível identificar apenas o período de taxa decrescente [10].

A evaporação da água dentro da estrutura dos materiais biológicos, é influenciada por vários fenômenos, como: capilaridade, ligação físico-química da água às substancias sólidas presentes no alimento, migração de sólidos, deformação do produto, entre outros; nota-se que tais fenômenos oferecem grande resistência ao processo de secagem [16].

Van Brackel resumiu e classificou em doze categorias, uma grande quantidade de curvas características de secagem que representam a taxa de secagem, de materiais publicados na literatura. Ele concluiu que, a categoria que representa os materiais biológicos é daqueles que apresentam apenas o período de taxa decrescente [17].

\subsection{Avaliação dos modelos de secagem}

Os dados de cinética de secagem da fibra do sisal, foram ajustados conforme os modelos empíricos de Page e Henderson, e os semiempíricos de Newton, Logarítmico e Midilli. Na Tabela 2, podemos observar os parâmetros dos modelos de cinética de secagem, que foram obtidos por meio de regressão não linear:

Tabela 2: Parâmetros dos modelos de Page, Henderson e Pabis, Newton, Logarítmico e Midilli para as temperaturas de secagem a $60{ }^{\circ} \mathrm{C}$ e $80^{\circ} \mathrm{C}$ em estufa com recirculação de ar.

\begin{tabular}{cccc}
\hline \multirow{2}{*}{ Designação dos modelos } & \multicolumn{2}{c}{ Parâmetros } \\
\cline { 2 - 4 } Page & $k\left(\mathrm{~min}^{-1}\right)$ & 0,010911 & $\mathbf{8 0}{ }^{\circ} \mathbf{C}$ \\
\hline \multirow{2}{*}{ Henderson e Pabis } & $n$ & 1,037502 & 0,014159 \\
& $k\left(\mathrm{~min}^{-1}\right)$ & 0,012890 & 1,092130 \\
Newton O'Callaghan & $a$ & 1,004249 & 0,020808 \\
\hline \multirow{2}{*}{ Logarítmico } & $k\left(\mathrm{~min}^{-1}\right)$ & 0,012817 & 0,020540 \\
& $k\left(\mathrm{~min}^{-1}\right)$ & 0,012282 & 0,019344 \\
& $a$ & 1,016966 & 1,038868 \\
Midilli & $b$ & 0,019264 & 0,028807 \\
& $k\left(\mathrm{~min}^{-1}\right)$ & 0,010010 & 0,013470 \\
& $a$ & 0,985378 & 0,987340 \\
& $b$ & 0,000028 & 0,000051 \\
& $n$ & 1,050714 & 1,097950 \\
\hline
\end{tabular}

Na Figuras 3, constata-se os modelos matemáticos de cinéticos de secagem ajustados aos dados experimentais de secagem para os materiais secos, em estufa com recirculação de ar, nas temperaturas de $60{ }^{\circ} \mathrm{C}$ e $80^{\circ} \mathrm{C}$. 


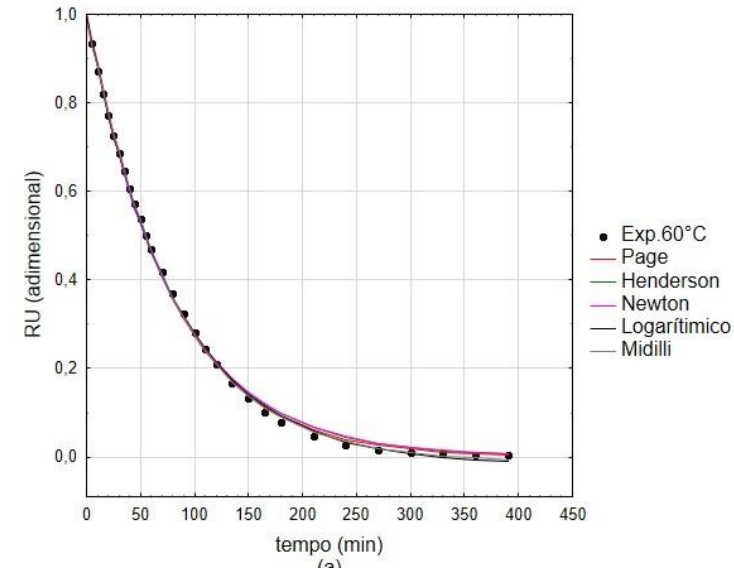

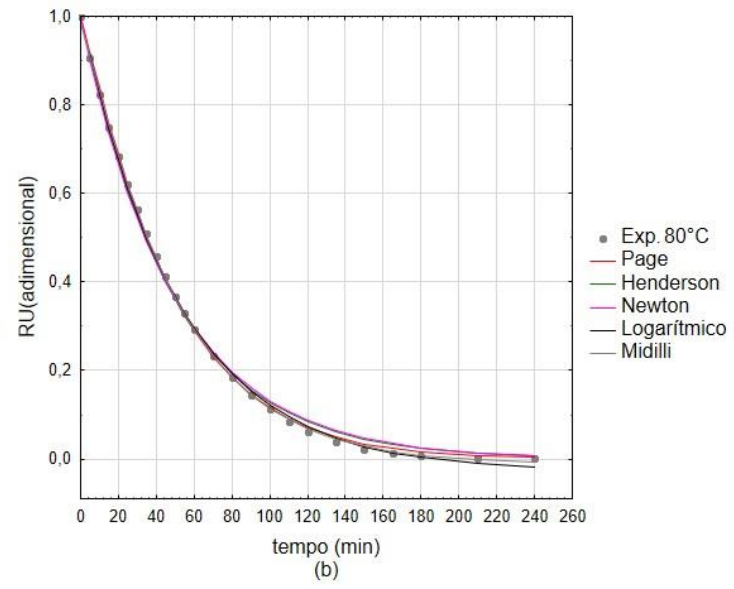

(b)

Figura 3: Ajuste dos modelos cinéticos de secagem aos dados experimentais de secagem a (a) $60{ }^{\circ} \mathrm{C}$ e (b) $80{ }^{\circ} \mathrm{C}$.

Por meio da Figura 3, observa-se que todos os modelos utilizados apresentam boa relação com os dados experimentais de secagem da fibra do sisal, em ambas as temperaturas avaliadas nessa pesquisa. Sendo assim, não é possível determinar qual o melhor modelo que representa os dados experimentais, apenas pela observação dos gráficos. É sugerido que a determinação do modelo seja feita com base nos maiores valores do coeficiente de determinação $\left(\mathrm{R}^{2}\right)$ e da razão do teste $F$, obtidos no teste estatístico de análise de variância (ANOVA).

A Tabela 3 apresenta os resultados do teste $F$ e o coeficiente de determinação $\left(\mathrm{R}^{2}\right)$, com significância de $5 \%$, obtidos no teste estatístico de análise de variância (ANOVA), para a comparação entre os cinco modelos ajustados aos dados experimentais de razão de umidade devido a secagem das fibras do sisal em diferentes condições de temperatura do ar.

Tabela 3: Análise de variância e coeficiente de determinação dos modelos dos modelos matemáticos da cinética de secagem do sisal em estufa de recirculação de ar nas temperaturas de $60^{\circ} \mathrm{C}$ e $80{ }^{\circ} \mathrm{C}$.

\begin{tabular}{ccccc}
\hline & \multicolumn{3}{c}{ Temperatura } \\
\cline { 2 - 5 } & \multicolumn{3}{c}{$\mathbf{6 0}^{\circ} \mathbf{C}$} & \multicolumn{3}{c}{$\mathbf{8 0}^{\circ} \mathbf{C}$} \\
\hline Designação do modelo & $\mathrm{F}_{\mathrm{ca}} / \mathrm{F}_{\mathrm{tab}}$ & $\mathrm{R}^{2}$ & $\mathrm{~F}_{\mathrm{cal}} / \mathrm{F}_{\mathrm{tab}}$ & $\mathrm{R}^{2}$ \\
Page & 13922,26 & 0,9996 & 11478,67 & 0,9997 \\
Henderson e Pabis & 9543,53 & 0,9994 & 3424,296 & 0,9989 \\
Newton O'Callaghan & 15458,23 & 0,9994 & 4689,68 & 0,9986 \\
Logarítmico & 14896,68 & 0,9997 & 6469,11 & 0,9996 \\
Midilli & 13466,95 & 0,9998 & 12044,46 & 0,9998 \\
\hline
\end{tabular}

Todos os modelos de cinética de secagem podem representar satisfatoriamente o fenômeno de secagem, por apresentarem coeficiente de determinação $\left(\mathrm{R}^{2}\right)$ superiores a $0,95 \%$. Entretanto, o modelo de Midilli se destaca, diante dos demais modelos para apresentar a cinética de secagem, por apresentar maiores valores de coeficiente de determinação em ambas as temperaturas de secagem.

O coeficiente de determinação $\left(\mathrm{R}^{2}\right)$ representa uma medida da quantidade de variância de uma variável que é explicada pela outra [18]. Segundo ARAUJO et al. [15], a utilização do coeficiente de determinação, como o único critério de avaliação para a escolha dos modelos de cinética de secagem, não constitui um bom parâmetro para representar um fenômeno de secagem, sendo necessário a análise conjunta de outros parâmetros estatísticos.

De acordo com NETO et al. [19] uma regra prática que podemos empregar é considerar a regressão como sendo útil para fins preditivos se o valor da razão entre $F_{c a} / F_{t a b}$ for, pelo menos, cerca de dez vezes maior que o valor do $F_{t a b}$. Podemos observar que a razão entre o $F_{c a l} / F_{t a b}$ apresenta valores muito altos, maiores que dez vezes o valor do $F_{t a b}$, mostrando que os modelos são uteis para fins preditivos. 


\subsection{Análise do planejamento fatorial $2^{3}$}

Com os dados obtidos no planejamento fatorial $2^{3}$, foi possível analisar os efeitos principais e suas interações entre fatores com seus respectivos erros padrões, para um nível de confiança de $95 \%$. A Tabela 4, apresenta a matriz de planejamento fatorial $2^{3}$.

Tabela 4: Matriz de planejamento fatorial $2^{3}$

\begin{tabular}{|c|c|c|c|c|c|}
\hline Experimento & concentração & TS & tempo & $q\left(m g \cdot g^{-1}\right)$ & Remoção \% \\
\hline 1 & -1 & -1 & -1 & 0,898 & 35,901 \\
\hline 2 & 1 & -1 & -1 & 2,768 & 55,360 \\
\hline 3 & -1 & 1 & -1 & 1,184 & 47,347 \\
\hline 4 & 1 & 1 & -1 & 2,997 & 59,938 \\
\hline 5 & -1 & -1 & 1 & 0,898 & 35,901 \\
\hline 6 & 1 & -1 & 1 & 2,882 & 57,649 \\
\hline 7 & -1 & 1 & 1 & 1,184 & 47,347 \\
\hline 8 & 1 & 1 & 1 & 3,169 & 63,372 \\
\hline 9 & -1 & -1 & -1 & 0,783 & 31,323 \\
\hline 10 & 1 & -1 & -1 & 2,825 & 56,504 \\
\hline 11 & -1 & 1 & -1 & 1,126 & 45,058 \\
\hline 12 & 1 & 1 & -1 & 2,997 & 59,938 \\
\hline 13 & -1 & -1 & 1 & 0,955 & 38,191 \\
\hline 14 & 1 & -1 & 1 & 3,054 & 61,083 \\
\hline 15 & -1 & 1 & 1 & 1,126 & 45,058 \\
\hline 16 & 1 & 1 & 1 & 3,054 & 61,083 \\
\hline 17 & -1 & -1 & -1 & 1,012 & 40,480 \\
\hline 18 & 1 & -1 & -1 & 2,711 & 54,215 \\
\hline 19 & -1 & 1 & -1 & 1,184 & 47,347 \\
\hline 20 & 1 & 1 & -1 & 3,054 & 61,083 \\
\hline 21 & -1 & -1 & 1 & 1,012 & 40,480 \\
\hline 22 & 1 & -1 & 1 & 2,940 & 58,794 \\
\hline 23 & -1 & 1 & 1 & 1,241 & 49,637 \\
\hline 24 & 1 & 1 & 1 & 3,226 & 64,517 \\
\hline
\end{tabular}

Na Tabela 4, pode-se observar que a maior quantidade de corante removida por unidade de adsorvente, foi obtida no experimento 24 , no qual as variáveis de controle estão em seus maiores níveis. A máxima quantidade de corante removida por unidade de adsorvente foi de $q=3,226 \mathrm{mg} \cdot \mathrm{g}^{-1} \mathrm{com}$ uma eficiência de Remoção $(\%)=64,52 \%$.

A Figura 4, apresenta o diagrama de Pareto para as variáveis resposta: a) $q$ (mg.g $\mathrm{g}^{-1}$ ) e b) Remoção (\%), para um nível de confiança de $95 \%$. 


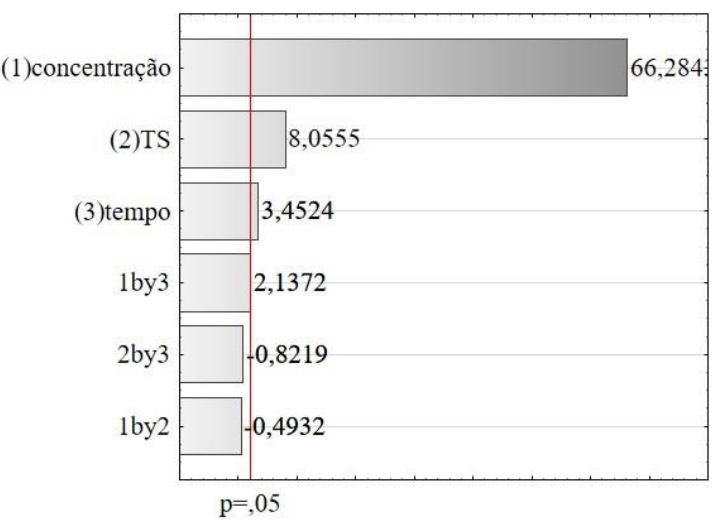

(a)

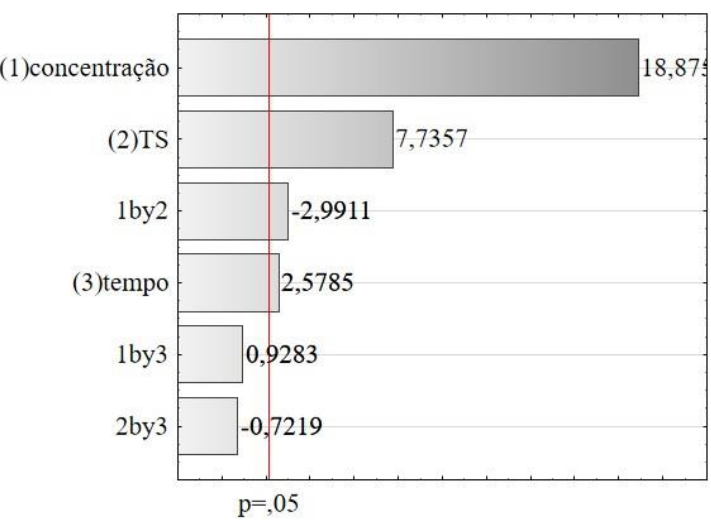

(b)

Figura 4: Diagrama de Pareto obtido por meio do planejamento fatorial $2^{3}$ para as variáveis resposta: (a) $q$ (mg. $\mathrm{g}^{-1}$ ) e (b) Remoção (\%).

Observa-se, pelo diagrama de Pareto, que as variáveis independentes concentração, temperatura de secagem $\left(\mathrm{TS}^{1}\right)$ e tempo, foram estatisticamente significativas para um nível de $95 \%$ de confiança.

Analisa-se que, no maior nível, as variáveis de controle afetam positivamente as respostas das variáveis dependentes $q\left(\mathrm{mg} . \mathrm{g}^{-1}\right)$ e Remoção $(\%)$, nas condições estudadas. Entretanto, a interação entre a concentração e a temperatura de secagem (1by2), fornece uma diminuição na resposta das variáveis dependentes.

Observa-se que a fibra do sisal seca, na temperatura de $80{ }^{\circ} \mathrm{C}$, proporcionou um aumento na capacidade de adsorção, isso é observado pelos valores obtidos pelas variáveis dependentes $q\left(\mathrm{mg}_{\mathrm{g}} \mathrm{g}^{-1}\right)$ e Remoção (\%). Segundo NASCIMENTO et al. [20] o aumento da temperatura pode produzir desobstruções de poros no interior da estrutura do adsorvente, permitindo que as moléculas maiores do corante possam penetrar no adsorvente.

Esse aumento na capacidade de adsorção do corante, pela fibra do sisal, pode estar associado com aumento nas áreas especificas da superfície das fibras. Segundo CANCIAM et al. [21] o aumento das áreas especificas pode estar associado ao aumento da temperatura de secagem, que gera uma diminuição na umidade presente no material, como também pode estar associado a uma certa rigidez dos sítios da parede celular do material. Com menos água adsorvida e uma estrutura de poro mais preservada do efeito de encolhimento, mais poros podem estar livres.

Pode-se observar que o aumento da concentração do corante de $25 \mathrm{mg} . \mathrm{L}^{-1}$ para $50 \mathrm{mg} . \mathrm{L}^{-1}$, proporcionou um aumento em $q$ (mg.g ${ }^{-1}$ ) e na Remoção (\%). Isso é explicado pelo fato de que em baixas concentrações, ainda existe uma grande quantidade de sítios disponíveis para que ocorra a adsorção [22]. $\mathrm{O}$ aumento da concentração, proporcionou uma maior distribuição das espécies, que compõem o corante, na superfície do adsorvente o que contribui para uma maior formação de ligações químicas na superfície [23].

MARCHI et al. [4] estudou a adsorção do corante básico azul de metileno por casca de Eucalyptuz grandis, e concluiu que as quantidades de corante adsorvida por grama de adsorvente foram mais elevadas em concentrações mais altas de corante.

\subsection{Construção dos modelos empíricos}

Partindo dos efeitos significativos, é possível construir os modelos empíricos para as variáveis dependentes $q$ (mg.g ${ }^{-1}$ ) e Remoção (\%). Essas equações consideram que a resposta varia linearmente com a variação dos fatores estudados em seus níveis. As Equações 5 e 6, apresentam esses modelos:

$$
q=2,011608+0,961460 * \mathrm{X}_{1}+0,116847 * \mathrm{X}_{2}+0,050077 * \mathrm{X}_{3}+0,031000 * \mathrm{X}_{1} * \mathrm{X}_{3}
$$

Rem. $\%=50,73365+8,72773 * \mathrm{X}_{1}+3,57694 * \mathrm{X}_{2}+1,19231 * \mathrm{X}_{3}+1,38308 * \mathrm{X}_{1} * \mathrm{X}_{2}$

\footnotetext{
${ }^{1}$ TS: Temperatura de secagem.
} 
onde: $q$, quantidade de corante adsorvida por unidade de massa de adsorvente utilizada (mg. $\left.{ }^{-1}\right) ;$ Rem. \%, eficiência na remoção do corante $(\%) ; X_{1}$, Concentração $\left(\mathrm{mg} . \mathrm{L}^{-1}\right) ; X_{2}$, Temperatura $\left({ }^{\circ} \mathrm{C}\right) ; X_{3}$, tempo (min).

Entretanto, para que o modelo proposto tenha significância estatística e possa ser utilizado em previsões, se faz necessário a aplicação de um teste estatístico de análise de variância (ANOVA), para que os modelos possam ser validos.

Os resultados do teste estatístico para os modelos que representam a quantidade de corante adsorvida por unidade de massa de adsorvente $(q)$ e a eficiência na remoção do corante (Rem. \%), são apresentados na Tabela 5 e 6 .

Tabela 5: Tabela ANOVA do planejamento fatorial $2^{3}$ para a variável dependente $q\left(\mathrm{mg} \cdot \mathrm{g}^{-1}\right)$.

\begin{tabular}{cccccc}
\hline Fonte & Soma Quadrática & $\begin{array}{c}\text { Gral de } \\
\text { Liberdade }\end{array}$ & $\begin{array}{c}\text { Média } \\
\text { Quadrática }\end{array}$ & $\mathbf{F}_{\text {cal }}$ & $\frac{\mathbf{F}_{\text {cal }}}{\mathbf{F}_{\text {tab }}}$ \\
\hline Regressão & 22,6013 & 6 & 3,7669 & 791,26 & 293,21 \\
Resíduos & 0,0809 & 17 & 0,0048 & & 0,006 \\
Falta de ajuste & 0,0001 & 1 & 0,0001 & 0,03 & \\
Erro puro & 0,0808 & 16 & 0,0051 & \\
Total & 22,6822 & 23 & & $\mathrm{~F}_{\text {tab }}=2,7$ \\
\hline
\end{tabular}

Tabela 6: Tabela ANOVA do planejamento fatorial $2^{3}$ para a variável dependente Remoção (\%).

\begin{tabular}{cccccc}
\hline Fonte & Soma Quadrática & $\begin{array}{c}\text { Gral de Li- } \\
\text { berdade }\end{array}$ & $\begin{array}{c}\text { Média } \\
\text { Quadrática }\end{array}$ & $\mathbf{F}_{\text {cal }}$ & $\frac{\mathbf{F}_{\text {cal }}}{\mathbf{F}_{\text {tab }}}$ \\
\hline Regressão & 2222,35 & 6 & 370,39 & 76,64 & 28,40 \\
Resíduos & 82,158 & 17 & 4,8328 & & 0,002 \\
Falta de ajuste & 0,055 & 1 & 0,0550 & 0,01 & \\
Erro puro & 82,103 & 16 & 5,1314 & \\
Total & 2304,51 & 23 & & $\mathrm{~F}_{\text {tab }}=2,7$ \\
\hline
\end{tabular}

É sugerido para que uma regressão possa ser considerada significativa, não apenas do ponto estatístico mas para que possa ser útil para fins preditivos, que o valor da razão $F_{c a l} / F_{t a b}$ para a regressão deve ser no mínimo dez vezes maior que o valor do $F$ tabelado, considerando os números apropriados de graus de liberdade, no nível de confiança escolhido [19]. Já para a falta de ajuste, em relação ao erro puro, a razão entre $F_{c a l} / F_{\text {tab }}$ deve apresentar o menor valor possível, pois um $F$ alto indica que existe uma grande falta de ajuste dos dados ao modelo obtido [24].

A análise estatística (ANOVA), para variável $q$, mostra que o modelo proposto possui significância estatística para um nível de confiança de $95 \%$. O modelo apresenta um coeficiente de determinação $\left(\mathrm{R}^{2}\right)$ alto, isso indica que o mesmo consegue explicar $99,64 \%$ dos dados de entrada. $\mathrm{O} F_{\text {cal }}$ apresenta um alto valor, maior que dez vezes o valor do $F_{t a b}$, indicado que o modelo é significativo do ponto de vista estatístico. $\mathrm{O}$ modelo também pode ser considerado como um modelo preditivo, pois a razão do teste $F$ para a falta de ajuste é menor que o $F_{t a b}$, indicando que o modelo se ajuste bem aos dados experimentais.

O modelo proposto para a variável Remoção (\%), também é considerado estatisticamente significativo. De acordo com o resultado obtido pelo coeficiente de determinação, pode-se afirmar que o modelo consegue representar $96,44 \%$ dos dados de entrada, e o valor da razão do teste $F$, referente a falta de ajuste, indica que o modelo proposto se ajusta bem aos dados experimentais da adsorção de corante têxtil sintético, podendo-se afirmar que o modelo pode ser utilizado para fins preditivos.

Os valores preditos e observados para a variável dependente $q\left(\mathrm{mg} \cdot \mathrm{g}^{-1}\right)$ e Remoção (\%) podem ser observados na Figura 5. 


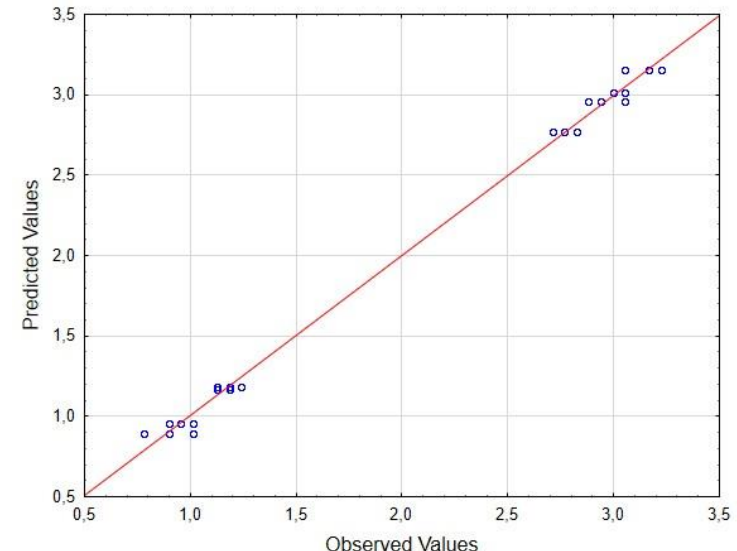

(a)

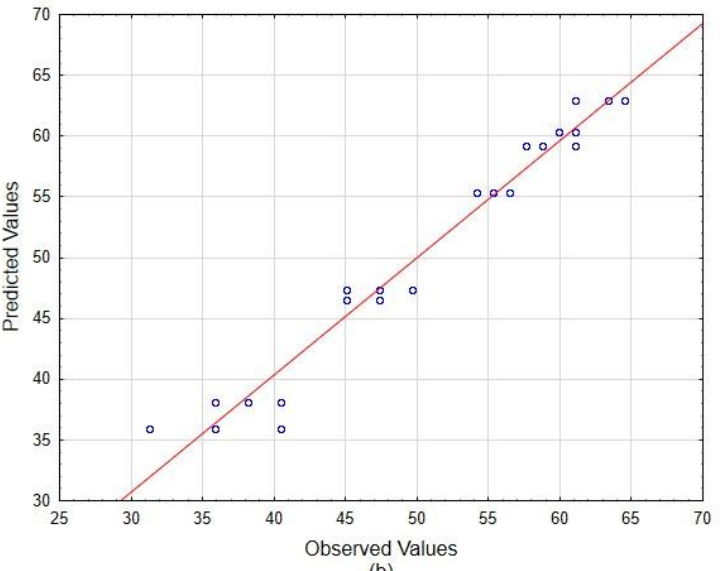

(b)

Figura 5: Valores preditos e observados para a variável resposta: (a) $q$ (mg.g-1) e (b) Remoção (\%) analisando as variáveis independentes, concentração, temperatura de secagem e tempo.

A Figura 5 mostra os valores preditos pelo modelo e os valores observados no experimento de adsorção do corante. Os valores preditos pelos modelos empíricos são representados pela reta vermelha e os dados observados são representados por pontos em azul.

Pode-se observar que os dados experimentais obtidos no planejamento experimental $2^{3}$, estão distribuídos bem próximos à reta vermelha, indicando que o modelo linear representa bem os dados experimentais de adsorção de corante sintético. Os dados obtidos no teste de análise de variância (ANOVA), corroboram essa afirmação.

Partindo das Equações 5 e 6, é possível construir os gráficos de contorno para a quantidade de corante removida $(q)$ e eficiência na remoção (Remoção \%). ção $(\%)$

As Figuras 6 e 7, apresentam os gráficos de contorno para as variáveis dependentes $q\left(\mathrm{mg} . \mathrm{g}^{-1}\right)$ e Remo-
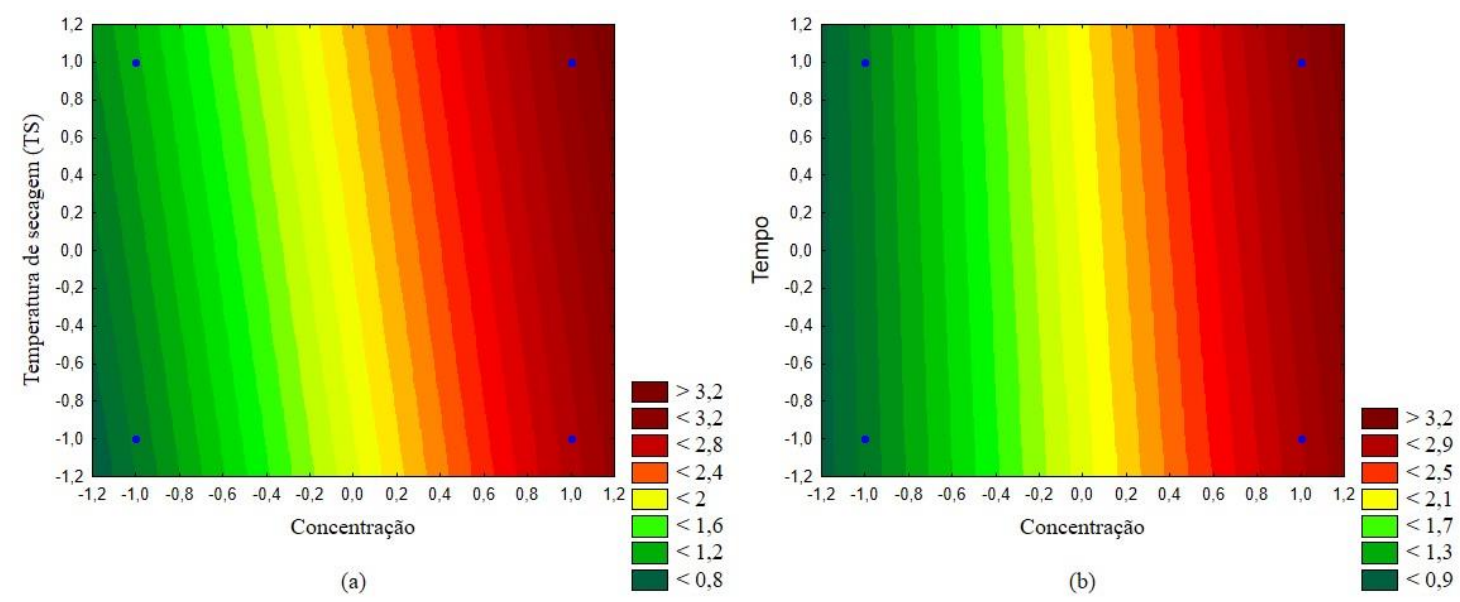

Figura 6: Gráfico de contorno para a variável resposta $q\left(\mathrm{mg} \cdot \mathrm{g}^{-1}\right)$ em função da: (a) temperatura versos concentração e (b) tempo versos concentração.

A partir da análise das Figuras 6 (a) e 6 (b), é possível observar a influência da temperatura de secagem e do tempo, sobre a quantidade de corante adsorvido por unidade de massa do adsorvente. Pode-se observar que os maiores valores de $q\left(\mathrm{mg} . \mathrm{g}^{-1}\right)$, são obtidos para o material seco em temperaturas mais elevadas, quando exposto a soluções com maior concentração. Isso é explicado pelo fato que o material seco em temperaturas mais elevadas, apresenta uma estrutura mais porosa, devido a eliminação de água presente na estrutura do material, o que favorece o processo de adsorção. Segundo CANCIAM et al. [21] o aumento da temperatura de secagem, de materiais lignocelulósicos, proporciona um aumento no volume dos poros, que é ocasionado pela perda de umidade. 
O mesmo comportamento se observa com relação ao tempo de contato, pois quanto maior for o tempo de contato, maior será a quantidade removida por unidade de massa do adsorvente.
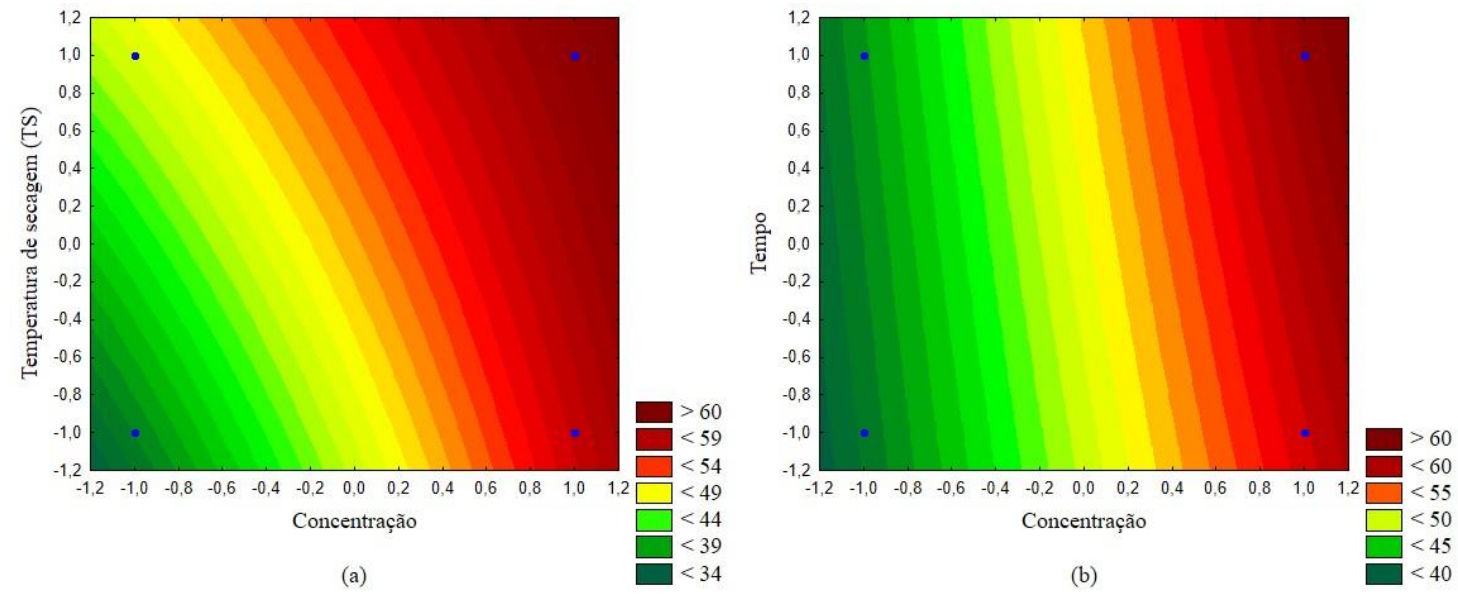

Figura 7: Gráfico de contorno para a variável resposta Remoção (\%) em função da: (a) temperatura versos concentração e (b) tempo versos concentração.

A partir da análise das Figuras 7 (a) e 7 (b), é possível observar a influência da temperatura de secagem e do tempo sobre a eficiência na remoção. Pode-se observar que os maiores valores para a variável Remoção (\%), são obtidos para o material seco a $80 \mathrm{C}$, quando exposto a soluções com maior concentração. Matérias secos em temperaturas mais elevadas apresentam maior porosidade que favorece o processo de adsorção. $\mathrm{O}$ mesmo comportamento se observa com relação ao tempo de contato, pois quanto maior for o tempo de contato, maior será o percentual de remoção.

\section{CONCLUSÕES}

A fibra do sisal in natura foi proposta como um material adsorvente de baixo custo para adsorção de corante sintético em solução aquosa. A cinética de secagem mais rápida foi obtida na temperatura de $80{ }^{\circ} \mathrm{C}$, sendo o modelo de Midilli o que melhor representou os dados de secagem em ambas as temperaturas. O planejamento fatorial $2^{3}$, mostrou que as variáveis de controle, concentração, temperatura de secagem e tempo, foram significativos do ponto de vista estático. Os gráficos de contorno mostram que os maiores valores de adsorção são obtidos quando as variáveis de controle estão em seus maiores níveis. Os maiores valores obtidos foram: $q=$ 3,226 mg.g $\mathrm{g}^{-1}$ e Remoção $(\%)=64,15 \%$.

Diante dos resultados obtidos, conclui-se que a fibra do sisal possui boas propriedades adsortivas podendo ser utilizado como adsorvente alternativo na remoção de corante têxtil sintético.

\section{BIBLIOGRAFIA}

[1] ZONATTI, W.F., AMARAL, M.C., GASI, F., et al., Reciclagem de resíduos do setor têxtil e confeccionista no Brasil: panorama e ações relacionadas. Sustentabilidade em Debate, Brasília, v. 6, n. 3, pp. 50-69, 2015.

[2] RIBEIRO, G.C.S.H., AGUIAR, C.R.L. Avaliação da adsorção do corante azul reativo CI 222 com adsorvente de resíduo sólido industrial seco e ativado quimicamente com H2SO4. In: Fórum internacional de resíduos sólidos. Porto Alegre, 2018.

[3] CASTRO, A. S., FRANCO, C.R., CIDADE, M.J.A. Adsorção de Corantes Azul Indosol, Laranja Indosol e Vermelho Drimaren em Solução Aquosa por Argila Branca. Resista Virtual de Química, Boa Vista, v. 10, n. 5, pp.1-14, 26 set. 2018.

[4] MARCHI, H.F., SOEIRO, T.N., HALASZ, M.R.T. Estudo da adsorção do corante básico azul de Metileno por cascas de eucalyptus grandis lixiviadas. In: XI Congresso Brasileiro de Engenharia Química em iniciação Científica, 2015, Campinas.

[5] BERTACCO, T.C.; CERON, L.P.; LIKS. L.A.S. Verificação da capacidade de adsorção da cinza da casca de arroz em efluente de cromo hexavalente em fluxo contínuo. Engevista, v. 21, n. 1, p. 114-125, fev. 2019. 
[6] SOBRINHO, O.P.L., SILVA, L.F.B., PEREIRA, Á.I.S., et al., Uma proposta de aula experimental utilizando mesocarpo de babaçu (Orbignya speciosa) na remoção do azul de metileno de soluções aquosas. Educación Química, México, p.314-318, 11 ago. 2015.

[7] BENDJEFFAL, H., DJEBLI, A., MAMINE, H. et al., Effect of the chelating agents on bio-sorption of hexavalent chromium using Agave sisalana fibers. Chinese Journal Of Chemical Engineering. China, pp. 984-992. 3 out. 2018.

[8] RODRIGUES, C., WOICIECHOWSKI, A.L., LETTI, L.A.J. et al., Materiais lignocelulósicos como matéria-prima para a obtenção de biomoléculas de valor comercial. Biotecnologia aplicada a agroindústria: fundamentos e aplicações. São Paulo: Brucher, 2016. E-book. ISBN 978-85-212-1115-0. Disponível em: https://openaccess.blucher.com.br/article-list/biotecnologia-aplicada-a-agro-e-industria-326/list\#undefined. Acesso em: 28 de set. 2019.

[9] GONELI, A.L.D., VIEIRA, M. C., VILHASANTI, H. C.B., et al., Modelagem matemática e difusividade efetiva de folhas de aroeira durante a secagem. Redalyc, v.44, n.1, p.56-64, fev. 2014.

[10] MENEZES, M.L., STRÖHER, A.P., PEREIRA, N.C., et al., de. Análise da cinética e ajustes de modelos matemáticos aos dados de secagem do bagaço do maracujá-amarelo. Engevista, Rio de Janeiro, v. 15, n. 2, p.176-186, 2013.

[11] PARK, K.J., ANTONIO, G.C., OLIVEIRA, R.A., et al., Conceitos de Processo Equipamentos de Secagem. Campinas, mar. 2007.

[12] ARAÚJO, C.S., MACEDO, L.L., VIMERCATI, W.C., et al., Cinética de secagem de acerola em leito de espuma e ajuste de modelos matemáticos. Brazilian Journal: Food Technology. Campinas, p. 1-9. 28 jan. 2017.

[13] BOTELHO, F.M., HOSCHER, R.H., HAUTH, M.R., et al., Cinética de secagem de grão de soja: Influência varietal. Revista Engenharia na Agricultura, Mato Groso, v. 26, n. 01, p. 13-25, 2018.

[14] LEITE, D.D. F., SANTOS, F.S., SANTOS, D.C., et al., Modelagem matemática da cinética de secagem da casca do abacaxi. Revista Verde de Agroecologia e Desenvolvimento Sustentável. Pombal - PB, v. 12, n. 4, p. 769-774, 2017.

[15] ARAUJO, W.D., GONELI, A.L.D., CORRÊA, P.C., et al., Modelagem matemática da secagem dos frutos de amendoim em camada delgada. Revista Ciência Agronômica, v. 48, n. 3, p. 448-457, jul-set, 2017.

[16] SANJINEZ-ARGANDONA, E.J., BRANCO, I.G., BITTENCOURT, T.U., et al., Influência da geometria e da temperatura na cinética de secagem de tomate (Lycopersicum esculentum). Cienc. Tecnol. Aliment., Campinas, p. 308-312, 2011.

[17] PARK, K.J.B., PARK. K.J., ALONSO, L.F.T. et al., Secagem: Fundamentação e equações. Revista Brasileira de Produtos Agroindustriais, Campina Grande, v. 16, n. 1, p. 93-127, 2014.

[18] FIELD, A. Descobrindo a estatística usando o SPSS. 2 ed. Porto Alegre: Artmed, 2009.

[19] NETO, B.B., SCARMINIO, I.S.; BRUNS, R.E. Como fazer experimento: pesquisa e desenvolvimento na ciência e na indústria. Campinas, SP: Editora da Unicamp, 2001.

[20] NASCIMENTO, R.F., LIMA, A.C.A., VIDAL, C.B., et al., Adsorção: aspectos teóricos e aplicações ambientais. Fortaleza: Imprensa Universitária - CE, 2014. Disponível em:

http://www.repositorio.ufc.br/bitstream/riufc/10267/1/2014_liv_rfdnascimento.pdf. Acesso em: 5 mar. 2018.

[21] CANCIAM, C.A., DIÓRIO, A., PEREIRA, N. C. Influência da temperatura de secagem nas propriedades texturais de alguns materiais lignocelulósicos: In. XXXVIII CONGRESSO BRASILEIRO DE SISTEMAS PARTICULADOS, Maringá PR, 2017.

[22] LEAL, P.V.B., GREGÓRIO, A.M., OTONI, E., et al., Estudo da adsorção do corante azul de metileno em resíduos de babaçu. Journal of Biotechnology and Biodiversity. v. 3, n. 4, p. 166 - 171, Nov. 2012.

[23] MATIAS, C.A., VILELA, P.B., PAULINO, A.T. Efeito da concentração inicial na adsorção de corante catiônico utilizando bráctea estéril de Araucaria angustifolia. In: Simpósio de Integração da Pós-Graduação Ciência, Técilogia e Inovação. Santa Catarina: SIGA, 2018.

[24] MACIEL, R.A., VIANA, C.M.C., SIMEÕES, M.G., et al., Desidratação osmótica de filés de Pirarucu (Arapaima gigas). Revista Brasileira de tecnologia Agroindustrial, Paraná, v. 06, n. 01, p.618-629, 2012. 
ORCID

Fellipe Farias Crispiniano

Cinthia Sany França Xavier

Fernando Fernandes Vieira

Marcelo Maia de Almeida https://orcid.org/0000-0003-1927-0740

https://orcid.org/0000-0002-4840-2653

https://orcid.org/0000-0002-1823-6236

https://orcid.org/0000-0001-7889-126X 